\title{
Family quality of life of South African families raising children with autism spectrum disorder
}

Schlebusch, L*, Dada, S.* \& Samuels, A.E.*\#

* Centre for Augmentative and Alternative Communication, University of Pretoria, Pretoria, Gauteng, South Africa

\# CHILD, School of Education and Communication, Jönköping University, Jönköping, Sweden

\begin{abstract}
This article describes the family quality of life among families who are raising a young child with autism spectrum disorder. Survey research was conducted with 180 families of children with autism spectrum disorder who were receiving disability-related services in the Gauteng province of South Africa. The principle measure used was the Beach Center Family Quality of Life Scale to assess five subdomains: family interaction, parenting, emotional well-being, material/physical well-being, and disability-related support. Results indicated that families felt the most satisfied with disability-related support and the least satisfied with the family's emotional well-being. Family income, family type, and the severity level of autism were significantly associated with how satisfied families felt about their quality of life.
\end{abstract}

Key words Appraisal of family income; autism; child characteristics; emotional well-being; family characteristics; unified theory of family quality of life 
The key role that families play throughout the lifespan of people with autism spectrum disorder (ASD) is increasingly acknowledged, and there is a growing interest in seeking positive approaches that can improve the well-being of these families (Gardiner \& Iarocci, 2012; Tint \& Weiss, 2015). Although family-focused ASD research has become more common, it remains underdeveloped (Cridland, Jones, Magee, \& Caputi, 2014), and critically so in low and middle-income countries like South Africa. Despite increased efforts to develop local capacity for support (Chambers et al., 2016; Grinker et al., 2012; Smith, MalcolmSmith, \& De Vries, 2016), South African families raising a child with ASD still experience significant challenges in accessing diagnostic, intervention, and educational services (De Vries, 2016; Malcolm-Smith, Hoogenhout, Ing, Thomas, \& De Vries, 2013). Disabilityrelated services, especially at the state level, are scarce and often heavily overburdened (Grinker et al., 2012; Van Biljon, Kritzinger, \& Geertsema, 2015). Families are mostly required to care for and support their children with ASD themselves, and to bear the cost of any professional services needed (De Vries, 2016). It is, therefore, essential to support and promote the well-being of these families since they are the primary support for children with ASD, often throughout their lifespan.

The family quality of life (FQOL) perspective provides a conceptual framework to seek positive approaches that can improve the quality of life of families raising a child with a disability. The outcome of this approach is families that are functioning optimally within their home and community, supporting the development of their children, and contributing to the ongoing stability of societies (Isaacs et al., 2007; Wang \& Brown, 2009). Supporting the FQOL of families in the early childhood years can potentially enhance a family's capabilities and skills to deal with adversities and challenges across their entire lifespan (Samuel, Rillotta, \& Brown, 2012). There has been considerable progress in the international research on FQOL 
in recent years, with a focus on conceptualisation, instrument development, explanatory research, and the examination of predictor variables on FQOL (Gardiner \& Iarocci, 2012). In more affluent countries, FQOL research is often used to inform policies and to evaluate services offered to families (Zuna, Turnbull, \& Summers, 2009). Conversely, in less resourced countries, the interest in FQOL arises more from a need to recognise the contributions of the family and to identify the service requirements of families who care for children and adults with disabilities (Ajuwon \& Brown, 2012).

While most of the FQOL research is based on studies of heterogeneous samples of children with intellectual and/or developmental disabilities, there is an increased focus on FQOL research on families of individuals with specific diagnoses, such as ASD (Eskow, Chasson, \& Summers, 2015; Eskow, Pineles, \& Summers, 2011; Gardiner \& Iarocci, 2015; McStay, Trembath, \& Dissanayake, 2014; Pozo, Sarriá, \& Brioso, 2013). Previous studies have reported that families of children with ASD fare much lower across a majority of quality of life domains compared to families of children with Down Syndrome (Brown, MacAdamCrisp, Wang, \& Iarocci, 2006), attention-deficit/hyperactivity disorder (Lee, Harrington, Louie, \& Newschaffer, 2008), and intellectual disability (Meral, Cavkaytar, Turnbull, \& Wang, 2013). This further supports the need to explore the concepts that influence the FQOL of families raising children with ASD. It is therefore both timely and necessary to investigate the FQOL of South African families who are raising a child with ASD.

FQOL is defined as the goodness of family life or the "conditions where the family's needs are met, and family members enjoy their life together as a family and have the chance to do things which are important to them" (Park et al., 2003: 368). It is conceptualised as a multidimensional construct that can be measured by indicators that are common to all 
families, with a focus to examine the perceptions and dynamics of the family unit as a whole (Zuna, Summers, Turnbull, Hu, \& Xu, 2010). It encompasses family satisfaction with both internal dynamics, such as cohesive family interactions, and external dynamics and resources, such as the availability of formal and informal supports (Gardiner \& Iarocci, 2015). Furthermore, the domains of FQOL (e.g., family interaction, parenting, emotional well-being, material/physical well-being) are considered to have universal properties that are assumptions or truths which are applicable to all families across cultures (Schalock \& Verdugo, 2014).

In an attempt to synthesise FQOL research, Zuna and colleagues (2010) proposed a unified theory of FQOL that seeks to explain what causes FQOL to vary among families of children with intellectual and other disabilities and to identify variables that positively impact a life of quality. The unified theory of FQOL proposes a model with four major explanatory concepts, namely: (a) systemic concepts (e.g., healthcare systems, policies), (b) performance concepts (e.g., formal support programmes and services), (c) family-unit concepts (i.e., family dynamics and family characteristics), and (d) individual family member concepts (i.e., characteristics, demographics, and beliefs). The theory proposes that these four concepts interact with each other. Singly or combined, these concepts predict a FQOL outcome, which then produces new family strengths, needs and priorities that re-enter the model as new input. This new input results in a continuous feedback loop throughout the life cycle of the family. However, the authors of the unified theory recognise that no single study could test the broad scope of this theory and recommend using the overarching FQOL theory as a framework for middle-range theories that can be translated into testable variables. The unified theory of FQOL therefore provides the big or global picture perspective, while individual studies provide the local knowledge to explain what causes FQOL to vary among families. 
The unified theory of FQOL distinguishes between dynamic family-unit concepts (i.e., aspects of interactions and ongoing relationships between two or more family members) and static family-unit concepts (i.e., traits or descriptors of the family as a whole) that directly influence FQOL (Zuna et al., 2010). Dynamic concepts are considered easier amenable to change to positively impact FQOL. For example, a study found that families who are raising young children with ASD who had a higher degree of regular family routines (i.e., a dynamic family-unit concept) also indicated a greater satisfaction of FQOL (Schlebusch, Samuels, \& Dada, 2016). However, an understanding of the static concepts (which are usually more difficult to manipulated) are also important, particularly in less resourced contexts (Zuna, Brown, \& Brown, 2014). While the experience of an individual disadvantage can create difficulties for families, experiencing multiple disadvantages can have an 'compounding effect' (McArthur \& Faragher, 2014). For example, the financial stress experienced by families can be further exuberated by the cost of having a child with a disability (Anderson, Dumont, Jacobs, \& Azzaria, 2007) and the potential loss of employment opportunities associated by caring for a child with a disability (Maul \& Singer, 2009). Findings of various studies suggest that family income and family employment have a significant association with FQOL (Hu, Wang, \& Fei, 2012; Mas et al., 2016; Meral et al., 2013; Wang et al., 2004) However, there are studies that did not find significant associations between family income and FQOL (Balcells-Balcells, Giné, Guàrdia-Olmos, \& Summers, 2011). Regarding family type, previous findings indicated that two-parent families indicate a higher FQOL compared to single-parent families (Gine et al., 2015; Mas et al., 2016).

When looking at the evidence concerning the influence of individual family member variables, research efforts have mainly focused on parents' educational level and age, while others have investigated the influence of the type of disability, the level of severity of 
disability, and age of the child on FQOL (Zuna et al., 2010). Various studies found that the severity level of the child's disability negatively influences FQOL (Balcells-Balcells et al., 2011; Hu et al., 2012; Schertz, Karni-Visel, Tamir, Genizi, \& Roth, 2016; Wang et al., 2004). However, there are studies that did not find significant associations between the level of severity of disability and FQOL (Gine et al., 2015). These contradictory findings are part of the beginning (but rapidly advancing field) of FQOL theory construction and the specification of potential predictor variables and FQOL-related outcomes that can positively impact FQOL (Schalock \& Verdugo, 2014).

The focus of the current paper is to measure and describe to the perceived FQOL of families raising a young child with ASD in South Africa. Additionally, the paper explores two of the postulations of the unified theory, namely that (a) family-unit concepts (particularly family characteristics such as the family income, family size, and family form), and (b) individual family member concepts (particularly characteristics of the parent, such the educational level, and characteristics of the child with ASD, such as the level of severity of ASD and the age of the child) serve as direct predictors of FQOL. Of interest to the international audience, this paper adds to the understanding of the unified FQOL theory by examining whether the selected family-unit and individual family member variables are associated with differences in the FQOL of families raising children with ASD. Locally, it recognises the needs and service requirements of these families and helps inform practitioners and policy makers on how to positively facilitate processes within the family and the family's environment to support and enhance the FQOL of these families. 


\section{Methods}

\section{Sampling and procedure}

Ethics approval for the study was obtained from the Research Committee of the University of Pretoria (Reference: 28524960) and the Gauteng Department of Education (Reference: D2015/239). Families could participate in the research study if they had a child younger than nine years old and diagnosed with ASD. The study specifically focused on families raising a young child with ASD. The study followed the age criteria set by UNESCO that defines early childhood as the period from birth to eight years old. However, the age criterion for this study was extended to under nine years old due to the difficulties of early access to disabilityrelated services in South Africa (Malcolm-Smith et al., 2013). The child furthermore had to attend disability-related schooling or intervention services in Gauteng, and the parent or caregiver had to be English literate. The requirement of attending disability-related services was to control for the influence of disability-related services on FQOL, as previous studies indicated a statistically significant difference between the FQOL of families of children with ASD receiving services compared to those still awaiting services (Eskow et al., 2015, 2011). Also, because of the diversity and unequal access to disability-related services across the country's nine provinces, only one South African province was included (Statistics South Africa, 2014). Gauteng is the smallest province; it is highly urbanised and represents the economic powerhouse of South Africa. It is the most populous province in South Africa with a population of nearly 12.3 million (Statistics South Africa, 2012). A total of 35 public and private organisations located in all five municipal districts of Gauteng identified 380 families. Each family received an envelope containing a survey booklet and information letter. The information letter explained the aims of the study and stated that participation was voluntary and based on informed consent, that the family's information would be kept confidential, and that they would not be identified in any manner. A parent or caregiver was asked to complete 
the survey on behalf of the family. Informed consent was obtained from all individual participants included in the study. Upon completion, the survey was returned to the organisation in a sealed envelope. A total of 180 families completed and returned the survey, resulting in a $47 \%$ return rate.

\section{Description of participants}

The way families were defined in this study allowed the researcher to have a broader understanding of the people who are closely involved in the day-to-day affairs of the household. Families reported other extended family members (such as grandparents, nieces, nephews, aunts, and siblings), close friends, and paid helpers as part of their family. This reflects the uniqueness of families in South Africa and underscores the importance of defining family broad enough to include the diversity of family members. As reported in Table 1, the participating families ranged from two-person bonds to large complex family systems. Regarding family characteristics, the majority of families were two-parent families (75\%), and most families had two children in the household (42\%). Families who had a grandparent or grandparents living with them were $18 \%$, while $22 \%$ of families had other family members or friends living with them, and $13 \%$ of families considered a paid helper to be part of their family. Families reported their total monthly income and also how they felt about their monthly family income. In terms of the families' appraisal of their monthly family income, only a few families felt they were financially well-off (4\%), and some felt they were managing well (9\%); the rest of the families felt they were doing okay (31\%), just getting by (35\%) or struggling $(21 \%)$. Regarding parent characteristics, the mean age of mothers was 36.70 years $(\mathrm{SD}=6.3)$, and that of fathers was 39.87 years $(\mathrm{SD}=6.4)$. The mean age of the oldest child was 9.60 years $(\mathrm{SD}=6.0)$, and the youngest child was 4.40 years $(\mathrm{SD}=2.3)$. The majority of the respondents were mothers $(68 \%)$. Sixty-six percent $(66 \%)$ of the respondents 
had a black ethnic background, $74 \%$ had a tertiary education, and $69 \%$ were employed fulltime. The average age of the children with ASD was 5.8 years $(\mathrm{SD}=1.38)$, with almost four times more boys $(81 \%)$ than girls $(19 \%)$. Based on a parental report, more than half of the children were described as having a moderate level of ASD (52\%), and 17\% of the children had co-occurring medical conditions or additional disabilities present.

Table 1. Participant Families Demographics $(\mathrm{N}=180$, unless otherwise stated)

\begin{tabular}{|c|c|c|}
\hline Description & $\mathbf{n}$ & $\%$ \\
\hline \multicolumn{3}{|l|}{ Family type } \\
\hline One-parent family & 36 & 20.0 \\
\hline Two-parent family & 135 & 75.0 \\
\hline Blended or stepfamily & 9 & 5.0 \\
\hline \multicolumn{3}{|l|}{ Number of children in the household $(n=178)$} \\
\hline One child & 51 & 28.7 \\
\hline Two children & 75 & 42.1 \\
\hline Three children & 34 & 19.1 \\
\hline Four or more children & 18 & 10.1 \\
\hline \multicolumn{3}{|c|}{ Families who have others living in the same household } \\
\hline Grandparents & 32 & 17.7 \\
\hline Related family members and/or friends & 40 & 22.2 \\
\hline Paid helper & 23 & 12.8 \\
\hline \multicolumn{3}{|l|}{ Monthly household income $(n=171)$} \\
\hline Less than R4 500 & 32 & 18.7 \\
\hline R4 501 - R12 500 & 26 & 15.2 \\
\hline R12 501 - R30 000 & 40 & 23.4 \\
\hline R30 001 - R52 000 & 35 & 20.5 \\
\hline More than R52 001 & 38 & 22.2 \\
\hline \multicolumn{3}{|c|}{ Families' appraisal of their monthly income $(n=171)$} \\
\hline Struggling & 36 & 21.1 \\
\hline Just getting by & 60 & 35.1 \\
\hline Doing okay & 53 & 31.0 \\
\hline Managing well & 15 & 8.8 \\
\hline Well off & 7 & 4.0 \\
\hline \multicolumn{3}{|c|}{ Educational background of parent/caregiver $(n=178)$} \\
\hline Grade 11 or less & 16 & 9.0 \\
\hline Grade 12 & 31 & 17.4 \\
\hline Diploma & 57 & 32.0 \\
\hline Bachelor's degree & 26 & 14.6 \\
\hline Postgraduate degree & 48 & 27.0 \\
\hline \multicolumn{3}{|c|}{ Employment status of parent/caregiver $(\mathrm{n}=178)$} \\
\hline Employed full-time & 124 & 69.1 \\
\hline Employed part-time & 13 & 7.3 \\
\hline Housewife & 15 & 8.4 \\
\hline Not currently working & 26 & 15.2 \\
\hline \multicolumn{3}{|l|}{ Gender of child with ASD $(n=178)$} \\
\hline Boy & 144 & 80.9 \\
\hline Girl & 34 & 19.1 \\
\hline \multicolumn{3}{|c|}{ Level of severity of ASD as rated by parents $(n=171)$} \\
\hline Mild & 60 & 35.1 \\
\hline Moderate & 88 & 51.5 \\
\hline Severe & 23 & 13.4 \\
\hline \multicolumn{3}{|c|}{ Co-occurring medical condition or additional disability present $(n=177)$} \\
\hline Yes & 30 & 16.9 \\
\hline No & 147 & 83.1 \\
\hline Age of family members & $(M)$ & $(S D)$ \\
\hline Age of father & 39.78 & 6.4 \\
\hline Age of mother & 36.70 & 6.3 \\
\hline Age of oldest child & 9.60 & 6.0 \\
\hline Age of youngest child & 4.40 & 2.3 \\
\hline Age of the child with ASD & 5.8 & 1.38 \\
\hline
\end{tabular}




\section{Measure}

There are no standardised or culturally validated measures available in South Africa to measure families' perceptions about their satisfaction of FQOL. Therefore, the Beach Center Family Quality of Life Scale (FQOL Scale), which was primarily developed for families of young children with disabilities living in the United States of America was utilised. This instrument is increasingly being used in other countries such as Spain (Balcells-Balcells et al., 2011; Verdugo, Córdoba, \& Gómez, 2005) and Hong Kong (Tait, Fung, Hu, Sweller, \& Wang, 2016), and for families of children with specific disabilities such as a hearing impairment (Jackson, Wegner, \& Turnbull, 2010) and for children with ASD (Eskow et al., 2011; Gardiner \& Iarocci, 2015). The FQOL Scale consists of 25 items. Parents respond to each item (e.g., "My family members show that they love and care for each other", "My family members have friends or others who provide support", "My family has a way to take care of our expenses") on a 5-point Likert scale of 1 (very dissatisfied) to 5 (very satisfied). The scores of the five subdomains are added to obtain a total score, which is then averaged into a single mean score. In the original study, the psychometric analysis provided support for a five-factor solution for the 25 questions: (a) family interaction, (b) parenting, (c) emotional well-being, (d) physical/material well-being, and (e) disability-related support. The internal consistency of the overall FQOL Scale was reported as a Cronbach coefficient alpha of .88, and the alphas for the subscales ranged from .74 to .90 (Hoffman, Marquis, Poston, Summers, \& Turnbull, 2006). The subscales of family interaction and physical/material well-being has demonstrated concurrent validity to other existing instruments (i.e., Family APGAR and Family Resource Scale; Hoffman et al., 2006).

The measurement of the FQOL concept across cultures require special care if the measure is transported from one culture to another (Schalock \& Verdugo, 2014). Due to the universal 
properties of the FQOL concept and the generic nature of the questions of the FQOL Scale, no changes were made to the original measure. It is, therefore, essential to report the reliability and validity results of the FQOL Scale in the current study in sufficient detail to demonstrate the reliability and validity of the FQOL Scale in the South African context. To determine the reliability of the FQOL Scale in the current study the Cronbach's alpha was calculated. The Cronbach's alpha for the overall FQOL Scale indicated excellent internal reliability (.92). The internal consistency for each of the five individual subscales of the FQOL Scale was: family interaction (.86), parenting (.80), emotional well-being (.74), physical/material well-being (.82), and disability-related support (.77). To determine the validity of the FQOL construct in the South African context, confirmatory factor analyses (CFA) were used to analyse the dimensional structure of the FQOL Scale. The proposed second-order five-factor model (Hoffman et al., 2006; Zuna et al., 2009b) was considered to verify whether the data obtained in the present study fit the original model. The CFA modeldata fit values included model-fit indices (assessing the goodness-of-fit) and factor loadings (assessing the parameter estimates). The overall fit indices of the CFA analysis revealed that the hypothesised second-order five-factor FQOL model offered a plausible fit to the data. Although the $\chi^{2}$ was relatively large and had a significant $p$-value, as is often the case with moderate to large samples, the $\chi^{2} / \mathrm{df}$ indicated a reasonable fit and the CFI of .85 indicated a suboptimal fit, while the RMSEA of .064 along with its $90 \%$ CI $[.064, .071]$ revealed a fair fit. The factor loadings (standardised regression weights) of the second-order FQOL model resulted in strong factor loadings of the measured variables. The standardised regression weights of the regression of the five latent constructs onto the second-order FOQL construct were: family interaction (.78), parenting (.93), emotional well-being (.80), physical/material well-being (.75), and disability-related support (.75). All the hypothesised factor loadings were statistically significant and sufficiently large (Harlow, 2014), and similar to previous 
results (Hoffman et al., 2006). This provided evidence that the construct of FQOL was measured in a reliable and valid manner for the study population and preliminary evidence about the suitability of the FQOL Scale in the South African context. The results provide further evidence about the universal properties of the FQOL construct.

\section{Analytic strategy}

Data were analysed using IBM SPSS version 22 and AMOS version 22. First, the reliability and validity of the FQOL Scale were determined, as reported in the measure section of this article. Thereafter, statistical analyses were conducted to (a) determine the descriptive statistics of the FQOL Scale; and (b) explore the potential associations between family-unit and individual family member variables and FQOL. Due to the nature of the demographic variables, a series of statistical analyses was undertaken to explore the relationships between variables. Independent t-tests were used to determine if there are statistically significant differences in the FQOL between two groups (e.g., families with boys with ASD and families with girls with ASD, or families with a working father and families without a working father). Cohen's $d$ was calculated to determine the effect size of the difference between the two groups and considered a small effect $(d=.2)$, medium effect $(d=.5)$, and large effect $(d$ $=.8)$ (Field, 2013). To explore the difference in FQOL of the four different employment groups, one-way ANOVA was conducted. Lastly, Spearman correlations were used to determine the associations between continuous demographic variables (e.g., the number of children in the household) and FQOL. The correlation coefficient is commonly used as a measure of the size of an effect, and it indicates the direction of the effect. A positive correlation coefficient indicates a positive relationship, while a negative correlation coefficient indicates a negative relationship. Values of around .1 indicate a small effect, 
values around .30 indicate a medium effect, and values around 0.50 indicate a large effect (Field, 2013).

\section{Results}

This section presents the results in relation to the study's objectives. First, the descriptive statistics of the families' perceived satisfaction with their FQOL are explained. Thereafter, the relationships between FQOL and a set of variables related to the characteristics of the family-unit, and the individual family member characteristics of the parent and the child with ASD are described.

Families' perceived satisfaction with their family quality of life

FQOL was scored on a scale of 1 (very dissatisfied) to 5 (very satisfied), meaning that higher scores indicate a greater satisfaction, while lower scores indicate a lesser satisfaction with FQOL (Table 2). The overall mean of the perceived satisfaction of the FQOL of the participating families was $3.83(\mathrm{SD}=0.61)$. Regarding the subdomains of FQOL, families felt the most satisfied with disability-related support $(M=4.03, \mathrm{SD}=0.715)$ and the least satisfied with their emotional well-being $(M=3.22, \mathrm{SD}=0.906)$.

Table 2. Satisfaction ratings of the overall family quality of life and the five subdomains.

\begin{tabular}{lrr}
\hline Description & Mean & SD \\
\hline Overall Family Quality of Life & 3.83 & 0.610 \\
Disability-related support & 4.03 & 0.715 \\
Family interaction & 4.01 & 0.723 \\
Physical/material well-being & 3.90 & 0.852 \\
Parenting & 3.89 & 0.657 \\
Emotional well-being & 3.22 & 0.906 \\
\hline
\end{tabular}

Note. Family Quality of Life was scored on a scale of 1 (very dissatisfied) to 5 (very satisfied).

\section{Characteristics of the family-unit and family quality of life}

The results of the independent $t$-tests indicate that there was only a statistically significant difference between the overall FQOL of one-parent families compared to two-parent families $(p<.05)$ (Table 3). Two-parent families had a higher overall FQOL $(M=3.91, \mathrm{SD}=0.567)$ 
compared to one-parent families $(M=3.55, \mathrm{SD}=0.661)$. This is considered a medium effect $(d=0.55)$. The remaining family variables (grandparents living with the family or not, other family members or friends living with the family or not, a paid helper living with the family or not) did not indicate statistically significant differences between the two groups $(p>.05)$.

Table 3. Independent t-tests to compare the differences of the overall family quality of life between two groups.

\begin{tabular}{|c|c|c|c|c|c|c|c|}
\hline Groups & $p$ & $\begin{array}{c}\text { Overall } \\
\text { FQOL } \\
(M)\end{array}$ & SD & $M-M$ & $d f$ & $t$ & $d$ \\
\hline \multicolumn{8}{|l|}{ CHARACTERISTICS OF THE FAMILY } \\
\hline 1. Two-parent family & .002 & 3.91 & 0.576 & 0.36 & 168 & 3.218 & 0.55 \\
\hline 2. One-parent family & & 3.55 & 0.661 & & & & \\
\hline 1. Grandparent(s) living with the family & .661 & 3.79 & 0.683 & -0.05 & 177 & -0.44 & 0.08 \\
\hline 2. Grandparent(s) not living with the family & & 3.84 & 0.595 & & & & \\
\hline $\begin{array}{l}\text { 1. Other family member(s) living with the } \\
\text { family }\end{array}$ & .163 & 3.71 & 0.641 & -0.16 & 177 & -1.40 & 0.24 \\
\hline $\begin{array}{l}\text { 2. Other family member(s) not living with the } \\
\text { family }\end{array}$ & & 3.87 & 0.600 & & & & \\
\hline 1. Paid helper living with the family & .913 & 3.82 & 0.449 & -0.01 & 177 & -0.109 & 0.02 \\
\hline 2. Paid helper not living with the family & & 3.83 & 0.631 & & & & \\
\hline \multicolumn{8}{|l|}{ CHARACTERISTICS OF THE PARENT } \\
\hline 1. Father working & .017 & 3.94 & 0.556 & 0.36 & 137 & 2.414 & 0.55 \\
\hline 2. Father not working & & 3.58 & 0.663 & & & & \\
\hline 1. Mother working & .495 & 3.85 & 0.614 & 1.76 & 171 & 0.684 & 0.11 \\
\hline 2. Mother not working & & 3.78 & 0.624 & & & & \\
\hline \multicolumn{8}{|l|}{ CHARACTERISTICS OF CHILD WITH ASD } \\
\hline 1. Boy with ASD & .823 & 3.83 & 0.625 & 0.02 & 175 & 0.223 & 0.04 \\
\hline 2. Girl with ASD & & 3.81 & 0.555 & & & & \\
\hline $\begin{array}{l}\text { 1. Additional disability or medical condition } \\
\text { present }\end{array}$ & .297 & 3.72 & 0.620 & -0.13 & 174 & -1.045 & 0.21 \\
\hline $\begin{array}{l}\text { 2. No additional disability or medical condition } \\
\text { present }\end{array}$ & & 3.85 & 0.611 & & & & \\
\hline
\end{tabular}

When considering the correlation between family variables and FQOL (Table 4), only the appraisal of the family income showed a statistically significant association with overall FQOL $(c=.445 ; p<.01)$. This relation had a positive direction, meaning that a higher family income is associated with higher level of FQOL. The correlation coefficient indicates that this is a medium effect. Both the reported monthly family income and the families' appraisal of the monthly income showed statistically significant, positive associations that are considered 
to be large effects with the physical/material well-being domain $(c=.515 ; p<.01 ; c=.589 ; p$ $<.01)$. Appraisal of the monthly household income also showed statistically significant, positive associations considered to be medium effects with the domains of emotional wellbeing and disability-related support $(c=.327 ; p<.01 ; c=.319 ; p<.01)$. For the remaining family variables (the age of the oldest child, age of the youngest child, and number of children in the household) there were no statistically significant relationships.

Table 4. Correlations between demographic variables and family quality of life.

\begin{tabular}{|c|c|c|c|c|c|c|}
\hline & $\begin{array}{c}\text { Family } \\
\text { Interaction }\end{array}$ & Parenting & $\begin{array}{l}\text { Emotional } \\
\text { Well-Being }\end{array}$ & $\begin{array}{c}\text { Physical/ } \\
\text { Material } \\
\text { Well-Being }\end{array}$ & $\begin{array}{l}\text { Disability- } \\
\text { related } \\
\text { Support } \\
\end{array}$ & $\begin{array}{l}\text { Overall } \\
\text { FQOL }\end{array}$ \\
\hline \multicolumn{7}{|c|}{ CHARACTERISTICS OF THE FAMILY } \\
\hline Monthly family income & .039 & .122 & .120 & $.515^{* * *}$ & $.181^{*}$ & $.266^{* *}$ \\
\hline Appraisal of this income & $.198^{* *}$ & $.287^{* *}$ & $.327^{* *}$ & $.589^{* * *}$ & $.319^{* * *}$ & $.445^{* *}$ \\
\hline Age of oldest child & -.031 & .077 & -.034 & $-.168^{*}$ & -.102 & -.054 \\
\hline Age of youngest child & -.085 & -.040 & -.018 & -.164 & -.114 & -.111 \\
\hline Number of children & -.013 & .089 & .022 & -.091 & -.098 & -.029 \\
\hline \multicolumn{7}{|c|}{ CHARACTERISTICS OF THE PARENT } \\
\hline Age of father & .052 & $.193^{*}$ & .028 & .140 & -.025 & .111 \\
\hline Age of mother & .030 & $.167^{*}$ & -.061 & .090 & -.066 & .026 \\
\hline Educational background & .069 & $.204^{* *}$ & .130 & $.402^{* *}$ & .128 & $.256^{* *}$ \\
\hline \multicolumn{7}{|c|}{ CHARACTERISTICS OF CHILD WITH ASD } \\
\hline Age & .076 & .109 & .083 & -.034 & .003 & .072 \\
\hline Level of severity of ASD & $-.243^{* *}$ & -.134 & $-.271^{* * *}$ & -.142 & $-.198^{* *}$ & $-.236^{* *}$ \\
\hline
\end{tabular}

\section{Characteristics of the parent and family quality of life}

For characteristics of the parent, the following variables were considered: employment status, father working, mother working, educational background, the age of the father, and the age of the mother. The four different type of employment groups were compared to determine if they indicated different overall FQOL scores. The groups were (a) parents who work fulltime, (b) parents who work part-time, (c) parents who are not currently working, and (d) homemakers. The analysis of variance showed that there were no statistically significant differences $(p=.107)$ between the overall FQOL of the four groups, $\mathrm{F}(3,172)=2.01$. Regarding fathers working or not, the results of the independent $t$-tests (Table 3 ) indicate that 
there was a statistically significant difference between families who had a working father and those who did not have a working father. Families who had a working father had a higher overall FQOL $(M=3.94, \mathrm{SD}=0.556)$ compared to families who did not have a working father $(M=3.58, \mathrm{SD}=0.663)$. This difference indicates a medium effect $(d=0.55)$. When evaluating the correlational analyses (Table 4), the parental education level showed statistically significant, positive associations with the parenting domain $(c=.204 ; p<.01)$, the material/physical well-being domain $(c=.4002 ; p<.01)$, and with the overall FQOL $(c=$ $.256 ; \mathrm{p}<.01)$. The relationship between parental education level and overall FQOL can be considered to have a small effect. For the remaining parent variables (i.e., age of the father and age of the mother), there were no statistically significant relationships.

\section{Characteristics of the child with ASD and family quality of life}

For the characteristics of the child with ASD, the following variables were considered: the gender of the child, the age of the child, the severity level of ASD, and whether the child had any additional disabilities or co-occurring medical conditions. As displayed in Table 3, there were no statistically significant differences between the FQOL of families who have boys with ASD and those who have girls with $\operatorname{ASD}(p>.05)$. This was the same for families with children who had additional disabilities or medical conditions present compared to those who did not have other disabilities or medical conditions present $(p>.05)$. When looking at the Spearman correlations reported in Table 4, the child's level of severity showed statistically significant, negative associations with the overall FQOL $(c=-.236 ; p<.01)$, as well as the subdomains of family interaction $(c=-.243 ; p<.01)$, emotional well-being $(c=-.271 ; p<$ $.01)$, and disability-related support $(c=-.198 ; p<.01)$. However, these associations indicated a small effect $(c<.03)$. 


\section{Discussion}

The first objective of this article was to describe the perceived FQOL of families raising a child with ASD in South Africa. The current study found that families were the most satisfied with the disability-related support that they are receiving. Compared to other studies that also measured the FQOL of families with children with ASD using the Beach Center FQOL Scale, the current study is the only one where families felt the most satisfied with this domain (Eskow et al., 2015, 2011; Gardiner \& Iarocci, 2015). In the other studies, families were most satisfied with the physical/material well-being. Considering that disability-related services for families are extremely limited in South Africa and that the sample for this study specifically included families who were receiving services, a possible explanation can be that these families felt grateful that they were receiving early intervention and/or educational services, and they, therefore, underscore the importance of these services. Studies have been conducted to compare the FQOL of families of children with ASD who were receiving disability-related services to families of children with ASD who were still awaiting services (Eskow et al., 2015, 2011). The results showed that those families who were receiving services indicated a higher level of satisfaction in all five subdomains as well as in their overall FQOL. In the South African context, it might be that children with disabilities who live in families who experience multiple disadvantages are more likely to have been excluded from receiving disability-related services (Fleisch, Shindler, \& Perry, 2012). As noted by Zuna and colleagues (2014), families in these situations may feel so overwhelmed and defeated that they do not even consider quality of life as a changeable construct. Therefore, a careful interpretation of this result is necessary.

Families reported that they were the least satisfied with their emotional well-being. This finding is particularly concerning as it echoes reports of other studies that also measured the 
FQOL of families with a child with ASD or other disabilities using the same measure (Eskow et al., 2011; Summers et al., 2007; Wang et al., 2006). The emotional support for families indicates a critical area of focus. Meral et al. (2013) refer to emotional support as the assistance in enhancing psychosocial functioning in terms of reducing stress and improving overall positive orientation to life. Services targeting the emotional well-being of families need to be developed or modified, and further research efforts need to be undertaken to understand how to support these families regarding their emotional well-being. As noted by Seligman and Darling (2007), all families need a little help from time to time. This is a normal aspect of family life and is met by most families in informal ways, with the help of family and friends. However, sometimes families' capabilities and informal support systems are insufficient to address their concerns, and professional help is required. Therefore, although the findings of this study emphasise the need for emotional support for families raising children with ASD, it is important to note that are research studies underscoring the positive functioning of families raising children with disabilities and that there are families who are coping well (Hastings, 2016).

The second objective was to depict the relationships between family-related variables and the families' perceived satisfaction with their FQOL. The influence of family income will be discussed first and then the influence of the family type on FQOL. Similar to other studies (Ferrer, Vilaseca, \& Olmos, 2016; Gardiner \& Iarocci, 2015; Wang et al., 2004), the current study also found that the family income had a direct, positive relationship with the families' perceived overall FQOL. However, in the current study, it was the family's appraisal of this income that revealed the more interesting finding. How families felt about their monthly income (i.e., struggling, just getting by, doing okay, managing well, or well off) showed more reliable relationships with the subdomains of FQOL and indicated a larger effect on the 
overall FQOL, compared to only considering the fiscal amount received. This finding might provide insight into the contradicting findings of previous studies, and highlights that understanding the economic hardship, beyond the total family income, allows for a deeper understanding of families' needs (Olsson \& Hwang, 2008). This finding is particularly relevant in the South African context. Among the major causes of poverty in South Africa is a lack of income. A national unemployment rate of $25.5 \%$ places a tremendous financial burden on many families (Statistics South Africa, 2014). Even when family members are gainfully employed, a history of intergenerational poverty means that in many traditional African families the income has to be shared amongst family members, thereby decreasing the amount of disposable income for disability-related services. It is critically important to address the economic viability of families. Financial satisfaction and stability can act as a buffer to other family challenges (Zuna et al., 2014). To help provide financial assistance to families with a child with a disability who are in need, the Social Assistance Act was amended in 2004 to remove the restriction that only children with severe disabilities requiring permanent homecare were eligible for a Care Dependency Grant. This was to also include families of children with mild to moderate disabilities in need of care and support services. However, investigations found that the families of children with mild to moderate disabilities were still economically excluded from the Care Dependency Grant (Proudlock, 2014). Pressure for the efficient implementation of this Act is, therefore, paramount for families raising a child with ASD in South Africa because receiving the Care Dependency Grant can decrease some of the economic stress of struggling families.

Regarding the family type, the current study found that two-parent families indicated a higher overall FQOL compared to single-parent families. This is similar to studies conducted in Spain (Gine et al., 2015; Mas et al., 2016). As noted by Giné et al. (2015), single parents of 
children with a disability can experience significant challenges because they are faced with the dual role of meeting emotional and financial needs of the entire family as well as with coping with the demands of caring for a child with a disability. This finding has particular implications for the South African context. To begin with, the proportion of fathers who are absent but living increased from 41.6\% to 47.4\% between 1996 and 2010 (Department of Social Development, 2012). This is a common and growing phenomenon that affects families in contemporary South Africa. More than $40 \%$ of all households in South Africa are headed by a single parent. An analysis of the characteristics of single parents in urban areas noted that the single parents were mostly African, female, and between the ages of 25 and 34 (Department of Social Development, 2012). It is, therefore, important to consider that the situation of single-parent families with a child with ASD might be different from that of families who have two parents living together while raising their child with ASD. It is also worthy to note that, although the differences were not statistically significant, the current study found that families who had extended family members, friends or a paid worker living with them indicated a slightly lower overall FQOL score. So, even though the support of others residing in the same house may have positive repercussions on sharing some of the parenting responsibilities (Schertz et al., 2016), it may also cause more stressful situations for parents and siblings, which can influence their FQOL. Therefore, the influence of the extended family on FQOL warrants further investigation.

The last objective was to identify relationships between individual family-member variables and FQOL. The parent's level of education, employment status and age, and the age, gender and the level of disability of the child with ASD were considered. Only the severity level of ASD indicated a reliable association with the families' overall FQOL, although this can be considered a small effect and should, therefore, be evaluated with caution. Previous findings 
by Pozo et al. (2013) indicated that the severity of ASD had a negative relation with FQOL for mothers, but had the opposite relation with FQOL for fathers. Gardiner and Iarocci (2015) did not find any significant relationship with the severity level of children with ASD and FQOL. However, it is important to consider that the level of severity of the child's ASD was based on parental report. Perceiving the child to have a severe level of disability can have a negative effect on the FQOL. This reflects the changing context of childhood disability, where the nature and severity of disability is not only a product of underlying medical conditions, but also a function of the demands, expectations, and social roles that children assume in their daily lives (Halfon, Houtrow, Larson, \& Newacheck, 2012).

\section{Limitations}

Although this paper is an important first step in understanding the FQOL of families who are raising children with ASD in South Africa, there are limitations that are worth noting, especially regarding the sample selection. First, this study is limited to only families of children with ASD who are receiving disability-related services. As noted, families who are not receiving any disability-related services may have a very different perspective about their FQOL. Second, the study focused on families of young children with ASD and does not represent the voices of those families with older children with ASD. Also, parents with low literacy levels and those who would have preferred to complete the survey in a different language might not have participated in the study. Although English is used in everyday life, education, business and politics, only a small minority of South Africans $(9,5 \%)$ speak English as their first language (Statistics South Africa, 2012). It is, therefore, important not to draw conclusions of this study beyond the study population. 


\section{Future research recommendations}

Future research should make a special effort to seek out and involve families who are not receiving any services yet, those who live in other provinces, and those who have a home language other than English, as they may be particularly isolated and may require very specific support. An important first step will be the translation, cultural adaptation and validation of a FQOL measure that can also be used by those with low literacy levels. As noted, further research efforts need to be undertaken to understand how to support the emotional well-being of families raising children with ASD. Also, the influence of the extended family on FQOL warrants further research investigation. Lastly, this research provides only a snapshot into FQOL at one point in time and from only one family member (mostly mothers). Although Wang et al. (2006) did not find statistically significant differences between the answers of mothers and fathers regarding their FQOL, future research that adopts a family life cycle perspective and that collects data from fathers and other family members could improve our understanding of FQOL.

\section{Practical implications}

The findings of this study paint a picture of the perceived FQOL of families with children with ASD and the broad range of factors that influence their FQOL. In particular, there are valuable implications for practitioners in South Africa that are potentially also applicable in other low-resource contexts. The preliminary evidence on the reliability and validity of the Beach Center FQOL Scale in the current study supports the universal properties of the FQOL concept and the suitability of using the FQOL Scale in the South African context. Practitioners can use this measure as a conversation starter to identify areas of family strengths and family needs. Family needs and service requirements can then be further prioritised, and help and support can be provided accordingly. While every family need 
identified through assessment may not be financially supported by a government agency, practitioners can devise creative ways to meet a larger number of families by restructuring how they deliver resources (Zuna et al., 2014). Practitioners should play a role in the development of lower-level interventions in the local settings, which is delivered by nonexpert facilitators or coaches (De Vries, 2016). For example, supportive practices such as family support groups or parent-delivered interventions might assist families in meeting their needs, especially their emotional needs (Zuna et al., 2014). In parent-delivered interventions, parents take an active role in delivering interventions in partnership with practitioners through advice, support, or direct training from practitioners (Zuna et al., 2014). Providing families with more cost-effective approaches to meet their support needs can be incredibly supportive of the family as a whole. Empowering parents with knowledge and skills can be of value to all families, regardless of the support need. Practitioners should also help families who need financial support to access available sources, for example the Care Dependency Grant to decrease some of the economic stress of struggling families. The findings of the current study also underscore the importance of finding out how families perceive the level of their child's disability. Lastly, practitioners should get to know who the family considers to be part of their family (e.g., grandparents or other family members living in the same household) and adopt a family-centred approach to ensure that parents or caregivers have adequate support in place. Practitioners must also be aware of the potentially different circumstances of single-parent families compared to two-parent families.

\section{Conclusion}

This study is the first to measure and describe the perceived FQOL of families raising a child with ASD in South Africa, thus contributing to the field of family-focused ASD research. The preliminary evidence on the universal properties of FQOL supports the adoption of a FQOL 
perspective to support the well-being of families raising children with ASD. Results indicate areas of strengths, such as the disability-related support that families are receiving, and also areas of family needs, particularly regarding their emotional well-being. The study supports two of the postulations of the unified theory of FQOL, namely that family-related factors (i.e., family income and family type) and individual family member factors (i.e., the severity level of a child's disability) are directly related to how families perceive their quality of life. Using a FQOL framework for children with ASD and their families provides a way for practitioners to think about and work towards what brings satisfaction and joy to families that they serve.

\section{Acknowledgements}

We gratefully acknowledge the families who kindly took the time to share some aspects of their family life in this study. We would also like to thank the organisations in Gauteng for devoting their time and expertise in the recruitment of participants.

\section{Funding}

This article was based on data collected for the doctoral dissertation of L.S supported in part by the University of Pretoria; and the National Research Foundation [UID: 88651]. The first author is currently supported by a University of Pretoria postdoctoral fellowship.

\section{Compliance with Ethical Standards}

All procedures performed in studies involving human participants were in accordance with the ethical standards of the institutional and/or national research committee and with the 1964 Helsinki declaration and its later amendments or comparable ethical standards. Ethics approval for the study was obtained from the University of Pretoria and the Gauteng Department of Education.

\section{Declaration of conflicting interests}

The authors declared no potential conflicts of interest with respect to the research, authorship, and/or publication of this article. 


\section{References}

Ajuwon, P. M., \& Brown, I. (2012). Family quality of life in Nigeria. Journal of Intellectual Disability Research : JIDR, 56(1), 61-70. http://doi.org/10.1111/j.13652788.2011.01487.x

Anderson, D., Dumont, S., Jacobs, P., \& Azzaria, L. (2007). The Personal Costs of Caring for a Child with a Disability: A Review of the Literature. Public Health Reports, 122(1), 316.

Balcells-Balcells, A., Giné, C., Guàrdia-Olmos, J., \& Summers, J. A. (2011). Family quality of life: Adaptation to Spanish population of several family support questionnaires. Journal of Intellectual Disability Research : JIDR, 55(12), 1151-1163. http://doi.org/10.1111/j.1365-2788.2010.01350.x

Brown, R. I., MacAdam-Crisp, J., Wang, M., \& Iarocci, G. (2006). Family quality of life when there is a child with a developmental disability. Journal of Policy and Practice in Intellectual Disabilities, 3(4), 238-245. http://doi.org/10.1111/j.17411130.2006.00085.x

Chambers, N. J., Wetherby, A. M., Stronach, S. T., Njongwe, N., Kauchali, S., \& Grinker, R. R. (2016). Early detection of autism spectrum disorder in young isiZulu-speaking children in South Africa. Autism, June. http://doi.org/10.1177/1362361316651196

Cridland, E. K., Jones, S. C., Magee, C. A., \& Caputi, P. (2014). Family-focused autism spectrum disorder research: A review of the utility of family systems approaches. Autism, 18(3), 213-222. http://doi.org/10.1177/1362361312472261

De Vries, P. J. (2016). Thinking globally to meet local needs. Current Opinion in Neurology, 29(2), 130-136. http://doi.org/10.1097/WCO.0000000000000297

Department of Social Development. (2012). White paper on families in South Africa. Department of Social Development. 
Eskow, K., Chasson, G. S., \& Summers, J. A. (2015). A cross-sectional cohort study of a large, statewide medicaid home and community-based services autism qaiver program. Journal of Autism \& Developmental Disorders, 45, 626-635. http://doi.org/10.1007/s10803-014-2217-4

Eskow, K., Pineles, L., \& Summers, J. A. (2011). Exploring the effect of autism waiver services on family outcomes. Journal of Policy and Practice in Intellectual Disabilities, 8(1), 28-35. http://doi.org/10.1111/j.1741-1130.2011.00284.x

Ferrer, F., Vilaseca, R., \& Olmos, J. G. (2016). Positive perceptions and perceived control in families with children with intellectual disabilities: relationship to family quality of life. Quality \& Quantity, February, 1-16. http://doi.org/10.1007/s11135-016-0318-1

Field, A. (2013). Discovering statistics using IBM SPSS statistics (4th ed.). London: SAGE Publications Inc.

Fleisch, B., Shindler, J., \& Perry, H. (2012). Who is out of school? Evidence from the Statistics South Africa Community Survey. International Journal of Educational Development, 32(4), 529-536. http://doi.org/10.1016/j.ijedudev.2010.05.002

Gardiner, E., \& Iarocci, G. (2012). Unhappy (and happy) in their own way: A developmental psychopathology perspective on quality of life for families living with developmental disability with and without autism. Research in Developmental Disabilities, 33(6), 2177-2192. http://doi.org/10.1016/j.ridd.2012.06.014

Gardiner, E., \& Iarocci, G. (2015). Family quality of life and ASD: The role of child adaptive functioning and behavior problems. Autism Research, 8(2), 199-213. http://doi.org/10.1002/aur.1442

Gine, C., Gracia, M., Vilaseca, R., Beltran, F. S., Balcells-Balcells, A., Montala, M. D., ... Mestre, J. M. M. (2015). Family quality of life for people with intellectual disabilities in Catalonia. Journal of Policy and Practice in Intellectual Disabilities, 12(4), 244-254. 
http://doi.org/10.1111/jppi.12134

Grinker, R. R., Chambers, N., Njongwe, N., Lagman, A. E., Whitney, G., Stronach, S., ... Wetherby, A. M. (2012). “Communities” in community engagement: Lessons learned from autism research in South Africa and South Korea. Autism Research, 5(3), 201-210. http://doi.org/10.1002/aur.1229.

Halfon, N., Houtrow, A., Larson, K., \& Newacheck, P. W. (2012). The changing landscape of disability in childhood. The Future of Children, 22(1), 13-42. http://doi.org/10.1353/foc.2012.0004

Hastings, R. P. (2016). Do Children With Intellectual and Developmental Disabilities Have a Negative Impact on Other Family Members? The Case for Rejecting a Negative Narrative. In R. M. Hodapp \& D. J. Fidler (Eds.), International Review of Research in Developmental Disabilities: Fifty years of Research in Intellectual and Developmental Disabilities (pp. 165-194). Elsevier Inc.

Hoffman, L., Marquis, J., Poston, D., Summers, J. A., \& Turnbull, A. (2006). Assessing family outcomes: Psychometric evaluation of the Beach Center Family Quality of Life Scale. Journal of Marriage and Family, 68(11), 1069-1083. http://doi.org/10.1111/j.1741-3737.2006.00314.x

Hu, X., Wang, M., \& Fei, X. (2012). Family quality of life of Chinese families of children with intellectual disabilities. Journal of Intellectual Disability Research : JIDR, 56(1), 30-44. http://doi.org/10.1111/j.1365-2788.2011.01391.x

Isaacs, B. J., Brown, I., Brown, R. I., Baum, N., Myerscough, T., Neikrug, S., ... Wang, M. (2007). The International Family Quality of Life Project: Goals and description of a survey tool. Journal of Policy and Practice in Intellectual Disabilities, 4(3), 177-185. http://doi.org/10.1111/j.1741-1130.2007.00116.x

Jackson, C. W., Wegner, J. R., \& Turnbull, A. P. (2010). Family quality of life following 
early identification of deafness. Language, Speech and Hearing Services in Schools, 41(4), 194-206. http://doi.org/10.1044/0161-1461(2009/07-0093)

Lee, L.-C., Harrington, R. A., Louie, B. B., \& Newschaffer, C. J. (2008). Children with autism: Quality of life and parental concerns. Journal of Autism and Developmental Disorders, 38(6), 1147-60. http://doi.org/10.1007/s10803-007-0491-0

Malcolm-Smith, S., Hoogenhout, M., Ing, N., Thomas, K. G., \& De Vries, P. (2013). Autism spectrum disorders - Global challenges and local opportunities. Journal of Child \& Adolescent Mental Health, 25(1), 1-5. http://doi.org/10.2989/17280583.2013.767804

Mas, J. M., Baqués, N., Balcells-Balcells, A., Dalmau, M., Giné, C., Gràcia, M., \& Vilaseca, R. (2016). Family quality of life for families in early intervention in Spain. Journal of Early Intervention, 38(1), 59-74. http://doi.org/10.1177/1053815116636885

Maul, C. A., \& Singer, G. H. S. (2009). “Just Good Different Things” Specific accommodations families make to positively adapt to their children with developmental disabilities. Topics in Early Childhood Special Education, 29(3), 155-170. http://doi.org/10.1177/0271121408328516

McArthur, M., \& Faragher, R. (2014). Children in families with complex needs. International Public Health Journal, 6(2), 199-211.

McStay, R. L., Trembath, D., \& Dissanayake, C. (2014). Stress and family quality of life in parents of children with autism spectrum disorder: Parent gender and the Double ABCX Model. Journal of Autism and Developmental Disorders, 44(12), 3101-3118. http://doi.org/10.1007/s10803-014-2178-7

Meral, B. F., Cavkaytar, A., Turnbull, A. P., \& Wang, M. (2013). Family quality of life of Turkish families who have children with intellectual disabilities and autism. Research and Practice for Persons with Severe Disabilities, 38(4), 233-246. http://doi.org/10.1177/154079691303800403 
Olsson, M. B., \& Hwang, C. P. (2008). Socioeconomic and psychological variables as risk and protective factors for parental well-being in families of children with intellectual disabilities. Journal of Intellectual Disability Research : JIDR, 52(12), 1102-1113. http://doi.org/doi:10.1111/j.1365-2788.2008.01081.x

Park, J., Hoffman, L., Marquis, J., Turnbull, A. P., Poston, D., Mannan, H., ... Nelson, L. L. (2003). Toward assessing family outcomes of service delivery: Validation of a Family Quality of Life Survey. Journal of Intellectual Disability Research : JIDR, 47(4/5), 367384. http://doi.org/10.1046/j.1365-2788.2003.00497.x

Pozo, P., Sarriá, E., \& Brioso, A. (2013). Family quality of life and psychological well-being in parents of children with autism spectrum disorders: a double ABCX model. Journal of Intellectual Disability Research : JIDR, 58(5), 442-458. http://doi.org/10.1111/jir.12042

Proudlock, P. (2014). South Africa's progress in realising children's rights: A law review. Cape Town: Children's Institute, University of Cape Town \& Save the Children South Africa.

Samuel, P. S., Rillotta, F., \& Brown, I. (2012). Review: The development of family quality of life concepts and measures. Journal of Intellectual Disability Research : JIDR, 56(1), 116. http://doi.org/10.1111/j.1365-2788.2011.01486.x

Schalock, R. L., \& Verdugo, M. A. (2014). Quality of life as a change agent. International Public Health Journal, 6(2), 105-117. http://doi.org/10.1111/j.1741-1130.2007.00135.x.

Schertz, M., Karni-Visel, Y., Tamir, A., Genizi, J., \& Roth, D. (2016). Family quality of life among families with a child who has a severe neurodevelopmental disability: Impact of family and child socio-demographic factors. Research in Developmental Disabilities, 53-54, 95-106. http://doi.org/10.1016/j.ridd.2015.11.028

Schlebusch, L., Samuels, A. E., \& Dada, S. (2016). South African families raising children 
with autism spectrum disorders: relationship between family routines, cognitive appraisal and family quality of life. Journal of Intellectual Disability Research : JIDR, 60(5), 412-423. http://doi.org/10.0.4.87/jir.12292

Seligman, M., \& Darling, R. B. (2007). Ordinary families, special children. (3rd ed.). New York: The Guildford Press.

Smith, L., Malcolm-Smith, S., \& de Vries, P. J. (2016). Translation and cultural appropriateness of the Autism Diagnostic Observation Schedule-2 in Afrikaans. Autism: The International Journal Of Research And Practice, May, 1-12. Retrieved from http://search.ebscohost.com/login.aspx?direct=true \&db=cmedm\&AN=27231336\&site= ehost-live \&scope $=$ site

Statistics South Africa. (2012). Census 2011 (Revised). Pretoria: Statistics South Africa. Retrieved from http://www.statssa.gov.za/publications/P03014/P030142011.pdf

Statistics South Africa. (2014). Census 2011: Profile of Persons with Disability in South Africa. Pretoria: Statistics South Africa. Retrieved from http://beta2.statssa.gov.za/publications/Report-03-01-59/Report-03-01-592011.pdf

Summers, J. A., Marquis, J., Mannan, H., Turnbull, A. P., Fleming, K., Poston, D. J., ... Kupzyk, K. (2007). Relationship of perceived adequacy of services, family-professional partnerships, and family quality of life in early childhood service programmes. International Journal of Disability, Development and Education, 54(3), 319-338. http://doi.org/10.1080/10349120701488848

Tait, K., Fung, F., Hu, A., Sweller, N., \& Wang, W. (2016). Understanding Hong Kong Chinese families' experiences of an Autism/ASD diagnosis. Journal of Autism and Developmental Disorders, 46(4), 1164-1183. http://doi.org/10.1007/s10803-015-2650-z Tint, A., \& Weiss, J. A. (2015). Family wellbeing of individuals with autism spectrum disorder: A scoping review. Autism. http://doi.org/10.1177/1362361315580442 
Van Biljon, S., Kritzinger, A., \& Geertsema, S. (2015). A retrospective case report on demographic changes of learners at a school for children with Autism Spectrum Disorders in the Gauteng Province. South African Journal of Childhood Education, 5(1), $42-61$.

Verdugo, M. A., Córdoba, L., \& Gómez, J. (2005). Spanish adaptation and validation of the Family Quality of Life Survey. Journal of Intellectual Disability Research : JIDR, 49(10), 794-8. http://doi.org/10.1111/j.1365-2788.2005.00754.x

Wang, M., \& Brown, R. (2009). Family quality of life: A framework for policy and social service provisions to support families of children with disabilities. Journal of Family Social Work, 12(2), 144-167. http://doi.org/10.1080/10522150902874842

Wang, M., Summers, J. A., Little, T., Turnbull, A., Poston, D., \& Mannan, H. (2006). Perspectives of fathers and mothers of children in early intervention programmes in assessing family quality of life. Journal of Intellectual Disability Research : JIDR, 50(12), 977-988. http://doi.org/10.1111/j.1365-2788.2006.00932.x

Wang, M., Turnbull, A. P., Summers, J. A., Little, T. D., Poston, D. J., Mannan, H., \& Turnbull, R. (2004). Severity of disability and income as predictors of parents' satisfaction with their family quality of life during early childhood years. Research and Practice for Persons with Severe Disabilities, 29(2), 82-94.

http://doi.org/10.2511/rpsd.29.2.82

Zuna, N. I., Brown, I., \& Brown, R. I. (2014). Family quality of life in intellectual and developmental disabilities : A support-based framework. International Public Health Journal, 6(2), 161-184.

Zuna, N. I., Selig, J. P., Summers, J. A., \& Turnbull, A. P. (2009). Confirmatory factor analysis of a Family Quality of Life Scale for families of kindergarten children without disabilities. Journal of Early Intervention, 31(2), 111-125. 
http://doi.org/10.1177/1053815108330369

Zuna, N., Summers, J. A., Turnbull, A. P., Hu, X., \& Xu, S. (2010). Theorizing about family quality of life. In Enhancing the quality of life of people with intellectual disabilities (Social Ind, pp. 241-276). Springer Science and Business Media. http://doi.org/10.1007/978-90-481-9650-0_15

Zuna, N., Turnbull, A., \& Summers, J. A. (2009). Family quality of life: Moving from measurement to application. Journal of Policy and Practice in Intellectual Disabilities, 6(1), 25-31. http://doi.org/10.1111/j.1741-1130.2008.00199.x

\section{Reference to published article:}

Schlebusch, L., Dada, S., \& Samuels, A. E. (2017). Family quality of life of South African families raising children with autism spectrum disorder. Journal of Autism and Developmental Disorders, 47, 1966-1977. Doi: 10.1007/s10803-017-3102-8 